\title{
Impact de la surveillance humaine sur les dégâts d'oiseaux en riziculture pluviale
}

\author{
Alphonse BOUET ${ }^{1^{*}}$, Arsène BOKA ${ }^{2}$ et Nazaire KOUASSI ${ }^{3}$ \\ ${ }^{\text {I}}$ Centre National de Recherche Agronomique (CNRA), BP 440 Man, Côte d'Ivoire. \\ ${ }^{2}$ Université NANGUI ABROGOUA, UFR des Sciences de la Nature, Abidjan, Côte d'Ivoire. \\ ${ }^{3}$ Centre National de Recherche Agronomique (CNRA), Laboratoire Central de Biotechnologies, \\ Adiopodoumé, Km 17, route de Dabou, 01 BP 1740 Abidjan 01, Côte d'Ivoire. \\ *Auteur correspondant ; E-mail : bouetalph@ gmail.com ; Tel : (225) 015010 93, (225) 07426485 , \\ (225) 55324343 ; Tél/fax :(225)32771700
}

\section{RESUME}

La production rizicole locale en Côte d'Ivoire est déficitaire de plus 900000 tonnes de riz blanchi à ce jour. Aussi, l'Etat importe depuis plusieurs années du riz afin de satisfaire la demande sociale. Les dégâts d'oiseaux constituent l'une des contraintes biotiques majeures à la base de cette faible productivité en riziculture. Traditionnellement, la surveillance humaine des rizières en début de maturation est le moyen de lutte anti aviaire utilisé par les riziculteurs. Mais cette pratique, souvent mal assurée, ne produit pas les résultats escomptés. Un essai a été conduit à la station de recherche de Man, en riziculture pluviale, dans le but de rendre plus efficace le gardiennage humain contre les oiseaux pendant la phase de maturation du riz. Quatre modalités de surveillance humaine contre les oiseaux ont été comparées, dans un dispositif matérialisé par quatre parcelles distantes les unes des autres de $50 \mathrm{~m}$. Les variables analysées sont : le nombre total de talles émises à la maturité technologique, le nombre de talles fertiles à la maturité technologique, le poids sec de la paille et le poids de paddy après séchage et vannage. Suite aux analyses statistiques, les résultats ont montré que la surveillance humaine d'une rizière de plateau pendant le cycle de croissance et de maturation de la panicule est nécessaire pour éviter ou réduire à plus de $80 \%$ les pertes de production induites par les oiseaux. Cependant, face aux exigences (présence permanente aux moments critiques des attaques d'oiseaux, insuffisance et /ou coût élevé de la main d'œuvre surveillant) liées au gardiennage, d'autres méthodes de lutte contre les oiseaux doivent être envisagées. Ces méthodes devront réduire voire se priver de la présence humaine pendant la période critique de la pression des oiseaux, c'est-à-dire de la phase de remplissage à la maturation des grains.

(C) 2014 International Formulae Group. All rights reserved.

Mots clés : Riziculture pluviale, surveillance humaine, oiseaux, Côte d'Ivoire, Man.

\section{INTRODUCTION}

Depuis plus de trente ans, la production locale de riz blanchi est déficitaire en Côte d'Ivoire (Anonyme 1, 2010; Anonyme 2, 2012). Ce déficit se chiffre aujourd'hui au-delà de $900000 \mathrm{t}$, (Anonyme 2, 2012) avec une incidence financière due à l'importation de plus 235 milliards francs CFA. Les dégâts causés par les oiseaux sont cités parmi les principales contraintes biotiques à la base de la faible productivité du riz. En effet, les oiseaux sont préjudiciables aux cultures de riz et autres céréales surtout, aussitôt après le semis et dès le stade laiteux 
des grains. Les pertes de récoltes ainsi induites varient de 10 à $15 \%$ en moyenne en Afrique subsaharienne (Manikowski et al., 1991) . Cependant, les pertes à la phase de maturation sont plus importantes et irréversibles. Il a été recensé 1100 espèces d'oiseaux en Afrique de l'Ouest. Parmi ceuxci, seulement $3.2 \%$ commettent en général des dommages aux cultures (Treca, 1990). Les plus préjudiciables étant l'oie de Gambie. le canard casqué, le tisserin gendarme, le tisserin à tête noire, le quéléa (travailleur à bec rouge), le Quelea erythrops (travailleur à tête rouge) et le moineau doré (Manikowski et al., 1991). Ces espèces aviaires engendrent des dégâts au semis et/ou durant la phase de maturation. L'espèce d'oiseau la plus populaire qui sévit aussi bien au semis qu'en période de maturation en riziculture est le quelea.

Plusieurs méthodes de lutte contre les oiseaux ont été mises au point (préventives, aménagement cultural, épouvantail, filets, la pulvérisation d'avicides par avion ou le dynamitage des dortoirs proches des cultures, Canon Tonnfort 5, cerf-volant, ballon «predator», méthodes olfactives (Anonyme 3, 2008 ; Treca, 1985 ; Treca, 1989 ; Hari et al., 1997), mais leur application, pour certaines, se heurte aux difficultés financières des exploitants et/ou à la nature des systèmes rizicoles pratiqués. En Côte d'Ivoire, non seulement le pouvoir financier des riziculteurs est faible, mais aussi deux systèmes rizicoles cohabitent. Il s'agit de la monoculture du riz fréquente en riziculture irriguée et le système de riziculture pluviale dans lequel le riz est généralement associé à d'autres cultures (vivrières et/ou de rente).

Dans un tel contexte agro-économique, il faut penser et mettre au point d'autres méthodes de lutte alternatives contre les oiseaux. Ces méthodes devront être relativement peu coûteuses au niveau financier et adaptées. Cette thématique liée aux oiseaux a fait l'objet de la présente étude qui a pour but de proposer une méthode de protection directe qui permette de réduire significativement les dégâts dus aux oiseaux en riziculture. La protection directe des cultures, selon Manikowski et al. (1991) sont des pratiques qui limitent l'accès des oiseaux aux champs cultivés.

\section{MATERIEL ET METHODES}

L'essai a été mis en place à la station de recherche de Man en condition de riziculture pluviale stricte. La variété de riz utilisée a été NERICA1 (appelée encore Bonfani, en ethnie Baoulé, pour son bon parfum) adaptée à la l'environnement pluvial de plateau. Quatre parcelles (traitements) de $300 \mathrm{~m}^{2}$ (20 m x $15 \mathrm{~m}$ ) chacune, représentant les modalités de surveillance contre les oiseaux ont été installées. Les quatre modalités définies sont :

- Pas de surveillance humaine du stade laiteux jusqu'à la maturité technologique du riz (SSLM) ;

- Surveillance humaine du stade laiteux au stade pâteux (SLP), de 6h30 le matin à 18h30 le soir ;

- Surveillance humaine du stade laiteux à la maturité technologique (SLM), de 6 h30 le matin à 18 h30 le soir ;

- Surveillance humaine du stade pâteux à la maturité technologique (SPM), de 6h30 le matin à $18 \mathrm{~h} 30$ le soir ;

Pour toutes ces modalités, les parcelles ont été surveillées contre les oiseaux, après le semis pendant sept (07) jours. Les traitements ont été isolés les uns des autres de $50 \mathrm{~m}$, afin de minimiser l'effet probable d'un traitement sur l'autre. A l'intérieur de chaque parcelle de traitement, 5 carrés de mesure d'un $\mathrm{m}^{2}$ ont été matérialisés sur une diagonale. Toutes les notations et mesures scientifiques ont eu lieu dans ces carrés.

Après le défrichement, puis déblaiement, un labour superficiel a été fait pour préparer le sol au semis. Le semis a été 
réalisé dès les premières pluies de juin, en poquets alignés séparés les uns des autres de 0,20 m. Ensuite, une palissade a été érigée autour de chacune des quatre parcelles (traitement) pour éviter d'éventuels biais dus à l'intrusion des animaux (agoutis). Trois sarclages manuels ont été effectués, avant le tallage, au tallage et en fin de tallage. Les paramètres mesurés ont été les suivantes:

- Nombre total de talles émises à la maturité technologique ;

- Nombre de talles fertiles à la maturité technologique ;

- Poids sec de la paille ;

- Poids de paddy après séchage et vannage.

\section{Analyse statistique}

Les variables mesurées ont été analysées, après estimation pour certaines, avec le logiciel GENSTAT. Les analyses de variance ont été exclusivement réalisées au seuil de 5\%. Les paramètres analysés étant : le nombre total de talles émises, le nombre de talles fertiles, le poids de paille sèche et le rendement en paddy.

\section{RESULTATS}

Effet de la période de surveillance humaine Sur le nombre de talles totales

Les résultats de l'analyse statistique, consignés dans le Tableau 1 révèlent un effet significatif de la modalité de surveillance sur la production de talles. Il est à noter cependant que le nombre de talles produites est plus élevé dans la parcelle non surveillée (SSLM) que dans les parcelles surveillées (SLM, SPM, SLP). Les valeurs affichées sont de 5 talles pour la parcelle non surveillée contre une moyenne de 3 talles dans les parcelles surveillées (Tableau 1). Mais ces valeurs n'obéissent pas à une cohérence liée à l'intérêt de la surveillance de la rizière contre les oiseaux.

\section{Sur le nombre de talles fertiles émises}

Le constat fait à partir du résultat de l'analyse de variance relative au nombre de talles fertiles est le même que celui portant sur le nombre de talles totales. En effet les talles fertiles produites sont plus nombreuses dans la parcelle non surveillée (3,75 talles) que dans les parcelles surveillées (Tableau 1). Une fois encore, les valeurs moyennes obtenues ne traduisent pas l'intérêt de la surveillance du champ contre les oiseaux.

\section{Sur le poids de paille sèche}

L'analyse statistique n'a montré aucun effet significatif de la modalité de surveillance (SLM, SPM, SPL, SSLM) sur le poids de la paille sèche (Tableau 1).

\section{Sur le rendement en paddy}

La surveillance contre les oiseaux a eu un effet significatif sur le rendement en paddy selon l'analyse statistique. Le rendement augmente en effet au fur et à mesure que la période de surveillance à partir du stade laiteux est longue. Cela est corroboré par les moyennes de rendement obtenues dans les parcelles surveillées 4 à 8 fois plus élevées $\left(1,072 \mathrm{t} \mathrm{ha}^{-1}\right.$ et $\left.0,970 \mathrm{t} \mathrm{ha}^{-1}\right)$ que dans les parcelles surveillées jusqu'au stade pâteux $\left(0,202 \mathrm{t} \mathrm{ha}^{-1}\right)$ et non surveillée $\left(0,132 \mathrm{t} \mathrm{ha}^{-1}\right.$; Tableau 1).

\section{Perte de production}

En considérant le rendement obtenu dans la parcelle surveillée du stade laiteux à la maturité technologique, comme le référentiel optimal, il ressort que les pertes de rendement dues aux oiseaux sous les modalités de surveillance varient de 0 à $90 \%$ (Tableau 1). Les fortes pertes ayant été enregistrées dans la parcelle surveillée jusqu'au stade pâteux $(80 \%)$ et la parcelle non surveillée $(90 \%)$. 
Tableau 1: Résultat de l'analyse de variance des variables rendement, nombre de talles totales, nombre de talles fertiles, poids de paille sèche et pourcentage de perte de rendement.

\begin{tabular}{|c|c|c|c|c|c|}
\hline \multirow[t]{2}{*}{ Traitements } & \multicolumn{4}{|c|}{ Variables analysées } & \multirow[b]{2}{*}{$\begin{array}{l}\text { Perte } \\
\text { de } \\
\text { rende } \\
\text { ment } \\
(\%)\end{array}$} \\
\hline & $\begin{array}{l}\text { Rendement } \\
\left(\mathrm{t} \mathrm{ha}^{-1}\right)\end{array}$ & $\begin{array}{l}\text { Nombre } \\
\text { de talles } \\
\text { totales }\end{array}$ & $\begin{array}{c}\text { Nombre } \\
\text { de talles } \\
\text { fertiles }\end{array}$ & $\begin{array}{l}\text { Poids } \\
\text { sec de } \\
\text { paille } \\
(\mathrm{kg})\end{array}$ & \\
\hline $\begin{array}{l}\text { Surveillance du stade } \\
\text { laiteux à la maturité } \\
\text { technologique (SLM) }\end{array}$ & $1,072 \mathrm{a}$ & $3,80 \mathrm{~b}$ & $3,498 \mathrm{a}$ & 0,405 & 0 \\
\hline $\begin{array}{l}\text { Surveillance du stade } \\
\text { pâteux à la maturité } \\
\text { technologique (SPM) }\end{array}$ & $0,970 \mathrm{a}$ & $3,27 b$ & $3,128 b$ & 0,179 & 8,3 \\
\hline $\begin{array}{l}\text { Surveillance du stade } \\
\text { laiteux au stade pâteux } \\
\text { (SLP) }\end{array}$ & $0,206 b$ & $3,74 b$ & $2,688 \mathrm{c}$ & 0,113 & 80 \\
\hline $\begin{array}{l}\text { Sans surveillance du } \\
\text { stade laiteux à la } \\
\text { maturité technologique } \\
(\mathrm{SSLM})\end{array}$ & $0,132 b$ & $5,33 \mathrm{a}$ & $3,756 a$ & 0,159 & 90 \\
\hline Moyenne générale & 0,595 & 4,04 & 3,267 & 0,214 & \\
\hline Probabilité & 0,007 & 0,015 & 0,002 & 0,205 & \\
\hline Lsd & 0,5926 & 1,202 & 0,4729 & 0,3013 & \\
\hline $\mathrm{CV}(\%)$ & 72,3 & 21,6 & 10,5 & 102,2 & \\
\hline Effet & HS & S & HS & NS & \\
\hline
\end{tabular}

\section{DISCUSSION}

Les résultats issus de la présente étude mettent en lumière trois informations majeures qui attirent l'attention. La première est que l'intérêt de la surveillance humaine d'une rizière contre les oiseaux n'est pas perçu en considérant la variables «nombre de talles émises ». En effet, la surveillance est assurée à partir du stade laiteux, après que la plante de riz ait exprimé son potentiel de tallage. Par ailleurs, au moment de la surveillance, les oiseaux sont intéressés exclusivement par la substance laiteuse contenue dans les glumelles et les grains pour leur alimentation. Ils ne pourraient par conséquent exercer un quelconque dégât structurel sur la plante de riz. Les différences notées entre les nombres de talles produites seraient dues entre autres à la fertilité physique et chimique des sols.

La deuxième information tirée des résultats est que la surveillance humaine du champ contre les oiseaux a un effet positif sur le rendement en paddy. Cette disposition permet d'éviter des pertes de production qui peuvent atteindre $90 \%$. Il a été rapporté dans certains pays des pertes de 50 à $100 \%$ de la production céréalière dus à l'oiseau Quelea, 
en absence de toute surveillance (Manikowski et al., 1991). L'efficacité de la surveillance humaine trouve son explication dans la protection de la cible d'attaque des oiseaux. En effet, dès le stade laiteux des grains de riz, les oiseaux se posent sur la panicule pour sucer la substance laiteuse contenue dans les glumelles ou prélèvent les grains pour leur alimentation. Les dégâts directs des oiseaux suceurs et granivores induisent aussi des dégâts indirects qui peuvent se traduire par la chute des étamines des talles secondaires dont certaines sont en floraison. Cette situation est encore plus prononcée avec la variété de riz NERICA1 dont la maturité est étalée dans le temps. Tous ces préjudices causés par les oiseaux ont pour conséquence la formation de grains vides ou vidés (pour non fécondation ou suçage des grains) et la réduction du nombre grains à maturité (par le prélèvement alimentaire des oiseaux granivores).

Le nombre et le poids des grains de riz par panicule étant des composantes majeures de rendement, les actions de déprédation directe et indirecte des oiseaux sur celles-ci conduisent finalement à la perte plus ou moins forte de production. Cette perte, dans les conditions de cette étude varie de 8,3 à $90 \%$. Au Sénégal, les pertes de production dues aux oiseaux varient de 30 à $70 \%$ selon (Gning, 2014).

La surveillance humaine d'une rizière à partir du stade laiteux ou pâteux à la maturité technologique est indispensable pour éviter de lourdes pertes de récolte dues aux oiseaux. Cependant, pour que la surveillance produise les effets satisfaisants, il faut que la vigilance et la présence effective aux moments cruciaux de la pression des oiseaux soient assurées. En effet, en riziculture pluviale de plateau, les oiseaux, tout genre confondu commencent leur quête alimentaire dès le lever du soleil pour rejoindre leurs refuges après le coucher du soleil. Il faut par ailleurs une ressource humaine suffisante pour mieux quadriller le champ. Selon notre étude, pour surveiller une rizière d'une superficie d'un hectare $\left(10000 \mathrm{~m}^{2}\right)$, il faudra solliciter au moins 10 personnes en considérant une personne pour $1000 \mathrm{~m}^{2}$. Certains travaux ont rapporté en effet que la surface qu'un gardien peut protéger efficacement ne doit pas dépasser un hectare. Ces mêmes sources plus optimistes préconisent, en cas d'une forte pression aviaire, les services d'au moins quatre gardiens par hectare (Manikowski et al., 1991).

En définitive, la surveillance humaine d'une rizière de plateau pendant le cycle de croissance et de maturation de la panicule est nécessaire pour éviter ou réduire à plus de $80 \%$ les pertes induites par les oiseaux. Cependant, il existe des contraintes liées à la surveillance humaine de la rizière. Il s'agit essentiellement de la discipline de surveillance, d'une éventuelle insuffisance de main d'œuvre et du coût financier de la surveillance. En effet, le coût de la main d'œuvre de surveillance équivaudrait à $80 \%$ de la valeur de la récolte selon des auteurs (Manikowski et al., 1991). Concernant la discipline, il est impératif que le surveillant soit présent pendant la période de forte pression, à savoir de $6 \mathrm{~h} 30$ ou $7 \mathrm{~h}$ du matin à $18 \mathrm{~h} 30$ le soir, notamment pour les grandes superficies. Face à toutes ces exigences liées au gardiennage, d'autres méthodes de lutte contre les oiseaux doivent être envisagées. Ces méthodes devront réduire voire se priver de la présence humaine pendant la période critique de la pression des oiseaux (du stade laiteux à la maturité technologique), c'est-àdire de la phase de remplissage des grains à la maturité.

\section{Conclusion}

La surveillance humaine disciplinée d'une rizière, en condition pluvial, du stade laiteux ou pâteux à la maturité des grains, permet d'éviter 8 à $90 \%$ des pertes de production dues aux oiseaux. Mais cela exige une ressource humaine très coûteuse, d'où, la nécessité de trouver d'autres méthodes de lutte anti-aviaire moins dépendante de la présence de l'homme au champ. 


\section{REFERENCES}

Anonyme 1. 2010. Programme de Productivité Agricole en Afrique de l'Ouest volet riz (PPAAO-1C) ; Projet de document de formulation de la Côte d'Ivoire. Ministère de l'Agriculture, Programme National Riz, République de Côte d'Ivoire, 36 p.

Anonyme 2. 2012. Stratégie nationale révisée de développement de la filière riz en Côte d'Ivoire (SNDR) 2012-2020. Office National de Développement de la Riziculture (ONDR), Ministère de l'Agriculture de Côte d'Ivoire, 40 p.

Anonyme 3. 2008: Protégez vos Cultures Contre les Dégâts des Oiseaux Ravageurs. Une Nouvelle Génération d'Epouvantails. Agriculture biologique des pays de la Loire, janvier 2008.

Gning O. 2014. Sénégal : entre 30 et $70 \%$ de production rizicole sénégalaise est détruite par les oiseaux. Agriculture, ruralité. Médiaterre; système d'information mondiale francophone pour le développement durable.
Manikowski S, N'diaye A, Treca B. 1991. Appui à la lutte anti aviaire. Manuel de protection des cultures contre les dégâts d'oiseaux. Projet TCP/SEN/005, FAO, $132 \mathrm{p}$.

Treca B. 1990. Description des principales espèces d'oiseaux d'eau potentiellement nuisibles aux cultures. Atelier de formation en lutte anti aviaire, 10-31 mai 1990, Niamey, NIGER, 5 pages.

Treca B. 1989. Les risques de dégâts d'oiseaux sur les rizières sahéliennes. ORSTOM, M’Bour, Sénégal, 09p.

Treca B. 1985. Les possibilités de lutte contre les oiseaux d'eau pour protéger les rizières en Afrique de l'ouest. $\mathrm{J} \quad \mathrm{o}$ m. d'Agaric. Trad. et de Bota. Appl. XXXII.

Hari KP, Dat VT, Trinh TT. 1997. Systèmes Améliorés de Riziculture

Pluviale. Division de la Production Végétale et de la Protection des Plantes. FAO : Rome. 